\title{
Evolutionary Game Based Access Control Protocol in Wireless Networks with Mobile Routers
}

\author{
Ippei AOKI $^{\dagger a)}$, Student Member, Koji YAMAMOTO ${ }^{\dagger}$, Member, Hidekazu MURATA ${ }^{\dagger}$, Senior Member, \\ and Susumu YOSHIDA ${ }^{\dagger}$, Fellow
}

\begin{abstract}
SUMMARY In existing systems of mobile routers, the frequency band is shared in uplinks from wireless terminals to mobile routers, and carrier sense multiple access with collision avoidance (CSMA/CA) is generally used as the medium access control protocol. To use the frequency band effectively, adaptive control is one promising approach. In this paper, a decentralized access control protocol in which mobile routers adaptively select the minimum contention window size is proposed. However, because of their mobility, which is one of the main difference between mobile routers and fixed access points, individual local area networks (LANs) consisting of the mobile routers and wireless terminals randomly interact with each other, and such random interactions can cause instability. To analyze the stability of the proposed control, evolutionary game theory is introduced because a system with random interactions between numerous decision-making entities can be analyzed by using evolutionary game theory. Using evolutionary game theory, the condition for existence of a convergence point is obtained. In addition, to implement the decentralized access control, a learning rule is proposed. In the proposed learning rule, each mobile router selects a strategy based on the result of past trials. From the simulation result, it is confirmed that the decentralized access control converges to a point closed to the stable state derived through evolutionary game theory.

key words: mobile router, evolutionary game theory, game theory, CSMA/CA
\end{abstract}

\section{Introduction}

In recent years, the number of studies on mobile routers has been increasing [1], [2]. Mobile routers are generally small devices, and they help in connecting wireless terminals to a base station of cellular networks [3], [4]. By connecting with a mobile router, wireless terminals do not need to access cellular networks directly. In general, the communication between mobile router and wireless terminals is based on the IEEE 802.11 protocol and the wireless terminals share the same frequency band because they use carrier sense multiple access with collision avoidance (CSMA/CA). When communicating with a mobile router, wireless terminals do not need to access cellular networks directly. Currently, the use of mobile routers is widespread and a local area network (LAN) can be constructed anywhere. In this paper, a LAN consisting of a mobile router and wireless terminals is referred to as a mobile LAN (MLAN).

The main difference between a mobile router and a fixed access point (AP) lies in their mobility. The mobil-

\footnotetext{
Manuscript received November 30, 2010.

Manuscript revised February 27, 2011.

${ }^{\dagger}$ The authors are with the Graduate School of Informatics, Kyoto University, 606-8501 Japan.

a) E-mail: aoki@hanase.kuee.kyoto-u.ac.jp

DOI: $10.1587 /$ transcom.E94.B.2225
}

ity of a mobile router gives rise to a new interesting phenomenon. Since mobile routers can be moved around and the wireless terminals share the same frequency band, there are often multiple MLANs in a closed area at the same time and these MLANs randomly interact with each other. For example, when the number of access requests from the wireless terminals in an MLAN is large, transmission opportunities in the other MLANs decrease. The essential point caused by the mobility is that two or multiple MLANs overlap spatially with each other.

To use the frequency band efficiently in a system where there are interactions among various MLANs, adaptive control is an promising approach. In this study, we focus on the access control protocol controlling contention window size in a decentralized way. Though it is possible to show the effectiveness of the access control protocol through a computer simulation in which various MLANs have numerous interactions, the simulation takes a long time and it is difficult to prove the validity of the results. Further, in a discussion on convergence, mathematical proof of convergence is necessary.

Using evolutionary game theory, the analysis of such numerous random interactions can be done easily. This theory can be used for analyzing the dynamics in a normalform non-cooperative game; the analysis can be performed more easily than simulation. A normal-form game consists of players, strategies, and utilities. Each player selects a strategy, and gets a utility as a result of interaction with other players. The primary solution concept for the normalform game is the Nash equilibrium. In a Nash equilibrium, no player has any motivation to deviate from his strategy because his utility decreases if he deviates. An evolutionarily stable strategy (ESS) is a solution in an evolutionary game and is related to a Nash equilibrium. Evolutionary game theory has been employed in biology to determine the mechanism of evolution [5], [6]. Recently, the number of researches involving evolutionary game theory is also increasing in the field of communication engineering [7], [8]. However, to the best of our knowledge, there are no studies on mobile routers that involve evolutionary game theory.

In this paper, evolutionary game-theoretic analysis of the interactions among MLANs is conducted. In the proposed system, the mobile routers adaptively select the minimum contention window size. Then, the proposed system is formulated as a game. In the game, multiple minimum contention window sizes are defined as strategies. From the 
analysis, we predict the conditions for convergence of the adaptive selection. In the proposed protocol, each mobile router selects the minimum contention window size and informs it to the wireless terminals in the same MLAN. Then, each wireless terminal transmits some packets using the minimum contention window size. Thus, the minimum contention window size selections of mobile routers are more important than the transmissions of wireless terminals. In this paper, minimum contention window size selections of mobile routers are mainly discussed. In addition, to implement the access control, a learning rule is necessary. In the learning rule, each player selects a strategy based on the utilities that the player has got. Then, the convergence quality and the stability are determined by using the learning rule. Without the learning rule, each mobile router selects a minimum contention window size randomly because there is no information to select a minimum contention window size. Evolutionary game theory is based on the learning. Thus, to implement our scheme, a learning rule is necessary.

There are many other backoff algorithms [9]-[13]. In [9]-[11], each AP controls contention window size of wireless terminals. In [12], each wireless terminal selects the optimal backoff time. Using this algorithm, the throughput is increased compared to the conventional CSMA/CA where the number of wireless terminals is large. In [13], contention window size is selected based on packet loss probability to reduce the convergence time of contention window size. Although these protocols are for fixed wireless LANs, it is not impossible to apply them to MLANs. In the proposed protocol, we adopt a similar approach. Thus, if all MLANs are identical and contention window size is selected from two values, performance of these conventional protocols and that of the proposed protocol are identical.

The rest of the present paper is organized as follows. In Sects. 2 and 3, the system model and the proposed protocol are presented. In Sect. 4, evolutionary game theory is discussed. In Sect. 5, the proposed scheme is evaluated with evolutionary game theory. In Sects. 6 and 7, the learning rules for the system are introduced and the evaluation of the dynamics is discussed. Finally, in Sect. 8, the conclusions are presented.

\section{System Model}

We consider the system model shown in Fig. 1. Mobile routers receive packets from wireless terminals in their own MLANs, and they transmit the packets to the base station. The link between the mobile router and the base station is controlled in a centralized way. Thus, packet collisions do not occur in this link. In this paper, we focus on the uplink from wireless terminals to mobile routers. We assume that numerous owners of MLANs move around, thus interacting randomly with each other. In such a situation, collisions can occur between packets from a single MLAN or between packets from different MLANs. This is because CSMA/CA is generally used as the medium access control protocol [3], [4]. For simplicity, it is assumed that the num-

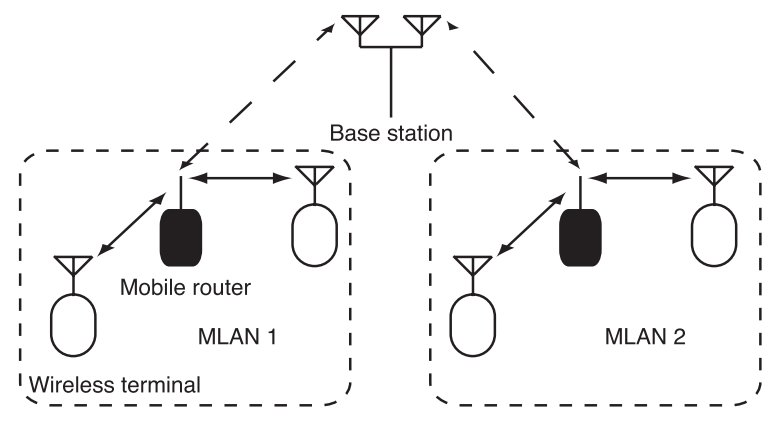

Fig. 1 System model consisting of two MLANs.

ber of MLANs that can be located in the same area and interact with each other is two. In other words, two MLANs are selected randomly from various MLANs and they interact with each other.

\section{Contention Window Size Control}

In this section, we explain conventional CSMA/CA and our proposed scheme. Then, we formulate the proposed scheme as a game.

\subsection{Conventional CSMA/CA}

CSMA/CA can be explained as follows [14]. There are assumptions as follows.

- Each wireless terminal always senses the channel during a backoff time.

- If a packet collision occur, the contention window size of the transmitting wireless terminals doubles and 1 is added to the contention window size.

- The maximum contention window size is predefined.

- The backoff time is decided on the basis of the contention window size.

- The initial contention window size is set to the minimum contention window size.

- If the backoff times of wireless terminals become 0 simultaneously, a packet collision occur.

Then, the processes are as follows.

1. If each wireless terminal has some packets to transmit, the wireless terminal decides the backoff time. Then, the wireless terminal starts to wait for the backoff time.

2. At the end of the waiting period, if the channel is not used, each wireless terminal starts to transmit packets to its AP. During the backoff time, if the channel has been used, each wireless terminal waits until the channel is not used and waits for the remaining backoff time.

3. The process then returns to step 1 .

The backoff time is given by

$$
B=R T_{\mathrm{s}},
$$

where $R$ is a random integer and $T_{\mathrm{s}}$ is the slot time. The 
range of $R$ is defined as

$$
0 \leq R \leq \mathrm{CW}
$$

where $\mathrm{CW}$ is the contention window size. The range of the contention window size is defined as

$$
\mathrm{CW}_{\min } \leq \mathrm{CW} \leq \mathrm{CW}_{\max }
$$

where $\mathrm{CW}_{\min }$ and $\mathrm{CW}_{\max }$ are the minimum and maximum contention window sizes, respectively.

\subsection{Proposed Scheme}

\subsubsection{Proposed Contention Window Size Selection}

Based on the conventional CSMA/CA, we propose that the mobile routers in the MLANs select $\mathrm{CW}_{\min }$ from two values, $\mathrm{CW}_{\text {min }}^{\mathrm{L}}$ and $\mathrm{CW}_{\text {min }}^{\mathrm{H}}$, where $\mathrm{CW}_{\text {min }}^{\mathrm{L}}<\mathrm{CW}_{\text {min }}^{\mathrm{H}}$. By using the proposed scheme, the number of packet collisions is expected to decrease compared to the scheme using only $\mathrm{CW}_{\text {min }}^{\mathrm{L}}$ because of the existence of mobile router using $\mathrm{CW}_{\min }^{\mathrm{H}}$. Further, it is also expected that the channel is used more efficiently than the protocol where only $\mathrm{CW}_{\min }^{\mathrm{H}}$ is used.

To implement the proposed scheme, there are four points that should be changed from the conventional scheme as follows.

- Each mobile router broadcasts $\mathrm{CW}_{\min }$ to the wireless terminals in the same MLAN.

- Wireless terminals change $\mathrm{CW}_{\text {min }}$ according to the information from the mobile router in the same MLAN.

- Mobile routers need to calculate the packet collision probability and channel efficiency.

- Mobile routers select $\mathrm{CW}_{\min }$ based on a learning rule.

The above four points are necessary to construct our scheme. The difficulty of the implementation is almost the same with the contention window size selection schemes in [9]-[11] that propose the access controls at each AP while theses schemes including our proposed scheme need the modification in wireless terminals. For example, in [9], adaptive contention window size selection at each AP is proposed. Since the approach of the proposed scheme is similar to that of [9], the computational complexity of the proposed scheme and that of [9] are almost the same.

\subsubsection{Game-Theoretic Formulation}

We formulate the system as a game as follows.

- Player: A set of mobile router and its wireless terminals in the same MLAN

- Strategy: Minimum contention window size $\mathrm{CW}_{\min } \in$ $\left\{\mathrm{CW}_{\min }^{\mathrm{L}}, \mathrm{CW}_{\min }^{\mathrm{H}}\right\}$

- Utility: Channel efficiency for each player and probability of packet collision of the wireless terminals in each MLAN

In the game-theoretic studies of ad-hoc networks, a pair of transmitter and receiver is often treated as a player as in [15], [16]. In addition, a mobile router and wireless terminals in the same MLAN generally belong to one owner. Therefore, it is reasonable that a set of mobile router and wireless terminals in the same MLAN is assumed to be a player.

The utility of a player with strategy $i$ interacting with other player with strategy $j$ is denoted as

$$
u(i, j)=e(i, j)-\alpha n(i, j),
$$

where $e(i, j)$ is the channel efficiency for the player with strategy $i$ and $n(i, j)$ is the probability of packet collision of own MLAN. The weight of $n(i, j)$ on $e(i, j)$ is denoted by $\alpha$. In this study, $e(i, j)$ is defined as

$$
e(i, j)=\frac{s(i, j)}{T_{\mathrm{a}}},
$$

where $s(i, j)$ denotes the number of successful transmissions when the intended mobile router chooses strategy $i$ and the opponent selects strategy $j$. In addition, this MLAN interacts with the MLAN where the mobile router selects strategy $j$. Further, $T_{\mathrm{a}}$ denotes a time period when two MLANs are in the same place.

It is reasonable to assume that the utility includes information on the packet collision probability. When a packet collision occurs, each wireless terminal has to reset the backoff time and the contention window size must be doubled. Therefore, transmission opportunities of each wireless terminal decrease. Thus, packet collisions decrease the utility of players.

\section{Evolutionary Game Theory}

Evolutionary game theory is used for analyzing the dynamics in normal-form games. In an evolutionary game, it is assumed that there are numerous players and that they randomly interact with each other. By these interactions, the players select the strategy that maximizes the expected utilities. Therefore, the ratio of the number of players having a common strategy to the total number of players converges to an equilibrium if some conditions are satisfied. In this section, we focus on the equilibrium in evolutionary game theory.

\subsection{Hawk-Dove Game}

In evolutionary game theory, the hawk-dove game is often used [5], [6]. In this game, there is a resource $V(>0)$, and players interact with each other for obtaining the resource. There are aggressive and passive strategies, which are referred to as hawk and dove, respectively.

The payoff matrix shown in Table 1 is drawn. When two players select a hawk, they fight. One player wins and the other loses. The player who wins the game gets $V$. The other player is said to get injured. Thus, it is assumed that losing is equal to losing a utility $C(>0)$. This means that 
Table 1 Payoff matrix in the hawk-dove game.

\begin{tabular}{|c|cc|}
\hline & hawk & dove \\
\hline hawk & $(V-C) / 2$ & $V$ \\
dove & 0 & $V / 2$ \\
\hline
\end{tabular}

$C$ is deduced from the loser side and $C$ is not moved to the winner side. In this game, the abilities of the players are assumed to be identical. Thus, the probability of winning is $1 / 2$ and the expected utility is $(V-C) / 2$. When two players select a hawk and a dove, the player with the hawk gets $V$. On the other hand, the player with the dove gets nothing and does not get injured. When two players select a dove, they share the resource; thus, each player gets $V / 2$.

This game corresponds to the game formulated in Sect. 3.2. The hawk corresponds to $\mathrm{CW}_{\min }^{\mathrm{L}}$. This is because wireless terminals of the MLAN where the mobile router selects $\mathrm{CW}_{\text {min }}^{\mathrm{L}}$ transmit more often than other wireless terminals. In a similar way, the dove corresponds to $\mathrm{CW}_{\text {min }}^{\mathrm{H}}$. Further, it can be considered that $V$ corresponds to the channel efficiency and $C$ corresponds to the packet collision probability.

\subsection{Evolutionary Stable Strategy (ESS)}

An ESS is a strategy that makes the state of the players evolutionarily stable (ES). In an ES state, the expected utilities of all the players are the same. If some players change strategies, the expected utilities are no longer the same. Subsequently, the other players select a strategy that increases their utilities. Thus, the state returns to the original state if the original state is ES.

First, we discuss an ESS in pure strategies. We consider pure strategies $I$ and $J(J \in\{1, \ldots, I-1, I+1, \ldots, M\})$, where $M$ is the number of strategies. It is assumed that strategy $I$ is an ESS and strategy $J$ is not an ESS. In the ES state, all players select strategy $I$. Then we assume that a few players change to strategy $J$. The ratio of the number of players with strategy $J$ to the total number of players is denoted by $p$. Therefore, the expected utility of the players with strategy $I$ is written as

$$
F(I)=F_{0}+(1-p) G(I, I)+p G(I, J),
$$

where $F_{0}$ denotes a utility that is independent of the strategies and $G(i, j)$ is the utility of a player with strategy $i$ interacting with a player with strategy $j$. On the other hand, the expected utility of strategy $J$ is written as

$$
F(J)=F_{0}+(1-p) G(J, I)+p G(J, J) .
$$

The condition for strategy $I$ to be the ESS is $F(I)>F(J)$. Since $p$ is very small, the condition can be written as

$$
G(I, I)>G(J, I), \quad \forall J .
$$

When $G(I, I)=G(J, I)$, the condition for strategy $I$ to be the ESS can be written as

$$
G(I, J)>G(J, J), \quad \forall J .
$$

Table 2 Payoff matrix in a general game $G(i, j)$.

\begin{tabular}{|l|l|ll|}
\hline \multicolumn{2}{|c|}{} & \multicolumn{2}{|c|}{$j$} \\
\cline { 3 - 4 } \multicolumn{2}{|c|}{} & 1 & 2 \\
\hline$i$ & 1 & $a$ & $b$ \\
& 2 & $c$ & $d$ \\
\hline
\end{tabular}

For example, in the hawk-dove game, a dove is not an ESS because $G$ (dove, dove) $<G$ (hawk, dove). On the other hand, a hawk is an ESS if $V>C$. This is because $G$ (hawk, hawk) $>G$ (dove, hawk) when $V>C$. An ESS in pure strategies is called a pure ESS.

Next, we discuss an ESS in mixed strategies; this ESS is called a mixed ESS. In a mixed strategy, a pure strategy is selected on the basis of its selection probability. We discuss the mixed ESS using the theorem of Bishop and Cannings [5]. The theorem is as follows [5]. It is assumed that each player selects a strategy from pure strategies $1,2, \ldots, M^{\prime}$ on the basis of its selection probability, where $M^{\prime}$ is the number of pure strategies. If a mixed strategy $I^{\prime}$ is an ESS in the game, then

$$
G\left(1, I^{\prime}\right)=G\left(2, I^{\prime}\right)=\cdots=G\left(M^{\prime}, I^{\prime}\right)=G\left(I^{\prime}, I^{\prime}\right) .
$$

We assume a general game where the payoff matrix is as given in Table 2 . We assume that strategies 1 and 2 are selected with probabilities $P$ and $1-P$, respectively, in mixed ESS $I^{\prime}$. From the expressions $G\left(1, I^{\prime}\right)=P a+(1-P) b$, $G\left(2, I^{\prime}\right)=P c+(1-P) d$, and $G\left(1, I^{\prime}\right)=G\left(2, I^{\prime}\right)$, we get

$$
P a+(1-P) b=P c+(1-P) d .
$$

Then, we get

$$
P=\frac{b-d}{c-a+b-d} .
$$

Similar to the case of the pure ESS, the condition for strategy $I^{\prime}$ to be a mixed ESS is $F\left(I^{\prime}\right)>F\left(J^{\prime}\right)$, where $J^{\prime} \in\{1,2\}$. Because $G\left(1, I^{\prime}\right)=G\left(2, I^{\prime}\right)=G\left(I^{\prime}, I^{\prime}\right)$, we can obtain

$$
G\left(I^{\prime}, J^{\prime}\right)>G\left(J^{\prime}, J^{\prime}\right), \quad \forall J^{\prime} .
$$

From this condition, the conditions for the existence of an ESS in the game can be obtained as follows:

$$
a<c, \quad d<b .
$$

For example, in the hawk-dove game, when $C>V$ and a hawk is selected with a probability $V / C$ in a mixed strategy, the mixed strategy is the ESS.

Next, we discuss the ES state. We consider a population consisting of numerous players that select strategies on the basis of their utilities. Moreover, we assume that in this case also the payoff matrix is also given by Table 2 . The ratio of the number of players with strategy 1 to the total population is denoted by $q$. Then, the ratio of the number of players with strategy 2 to the total population is denoted by $1-q$. In the ES state, the expected utilities are the same. Therefore, for the ES state, we can obtain the following equation: $q a+(1-q) b=q c+(1-q) d$. From this equation, we obtain 


$$
q=\frac{b-d}{c-a+b-d}=P .
$$

Hence, it is proved that the state is ES if the mixed strategy with a probability $P$ is a mixed ESS and the number of the pure strategies is two [5].

\section{Equilibrium Evaluation}

We evaluate the protocol described in Sect. 3 by performing a computer simulation and analyzing with the help of evolutionary game theory. The simulation parameters are listed in Table 3. All the wireless terminals are assumed to always have packets to transmit. For simplicity, it is assumed that the wireless terminals can perfectly detect whether the channel is idle or not.

Table 4 shows the channel efficiency $e(i, j)$ as a result of simulation. As is apparent from the table, $e\left(\mathrm{CW}_{\min }^{\mathrm{L}}, \mathrm{CW}_{\min }^{\mathrm{H}}\right)$ is the maximum. This is reasonable because the wireless terminals with $\mathrm{CW}_{\text {min }}^{\mathrm{L}}$ transmit packets more often than those with $\mathrm{CW}_{\min }^{\mathrm{H}}$. As shown in Table $4, e\left(\mathrm{CW}_{\min }^{\mathrm{H}}, \mathrm{CW}_{\min }^{\mathrm{L}}\right)$ is the minimum. This result indicates that the wireless terminals with $\mathrm{CW}_{\text {min }}^{\mathrm{L}}$ often transmit packets. Therefore, the transmission opportunities for wireless terminals with $\mathrm{CW}_{\min }^{\mathrm{H}}$ decrease.

Table 5 shows the packet collision probability $n(i, j)$. The results are reasonable because a decrease in the minimum contention window size increases the packet collision probability.

Table 6 shows the utility of a player when $\alpha=3$. From the result and (14), the condition for the existence of a mixed ESS in the game is satisfied. Moreover, from Table 6 and

Table 3 Simulation parameters.

\begin{tabular}{c|c}
\hline Parameters & Values \\
\hline Number of wireless terminals per MLAN & 5 \\
$\mathrm{CW}_{\max }$ & 1023 \\
$\mathrm{CW}_{\min }^{\mathrm{L}}$ & 15 \\
$\mathrm{CW}_{\min }^{\mathrm{H}}$ & 127 \\
$T_{\mathrm{s}}$ & 1 \\
$T_{\mathrm{a}}$ & 10000 \\
Number of examinations & 20000 \\
\hline
\end{tabular}

Table 4 Channel efficiency $e(i, j)$.

\begin{tabular}{|c|c|cc|}
\hline \multicolumn{2}{|c|}{} & \multicolumn{2}{|c|}{$j$} \\
\cline { 3 - 4 } & $\mathrm{CW}_{\text {min }}^{\mathrm{L}}$ & $\mathrm{CW}_{\text {min }}^{\mathrm{H}}$ \\
\hline \multirow{2}{*}{$i$} & $\mathrm{CW}_{\min }^{\mathrm{L}}$ & 0.12 & 0.21 \\
& $\mathrm{CW}_{\min }^{\mathrm{H}}$ & 0.018 & 0.054 \\
\hline
\end{tabular}

Table 5 Packet collision probability $n(i, j)$.

\begin{tabular}{|c|c|cc|}
\hline \multicolumn{2}{|c|}{} & \multicolumn{2}{|c|}{} \\
\cline { 3 - 4 }$i$ & $\mathrm{CW}_{\text {min }}^{\mathrm{L}}$ & $\mathrm{CW}_{\text {min }}^{\mathrm{H}}$ \\
\hline \multirow{2}{*}{$i$} & $\mathrm{CW}_{\min }^{\mathrm{L}}$ & 0.051 & 0.044 \\
& $\mathrm{CW}_{\text {min }}^{\mathrm{H}}$ & 0.0093 & 0.0054 \\
\hline
\end{tabular}

(15), the ratio of the number of players with $\mathrm{CW}_{\text {min }}^{\mathrm{L}}$ to the total number of players in the ES state, $r^{\star}$, is found to be

$$
r^{\star}=\frac{0.0079-0.038}{[(-0.0096)-(-0.031)]+0.0079-0.038}=0.66 \text {. }
$$

This means that $66 \%$ of all the players select $\mathrm{CW}_{\text {min }}^{\mathrm{L}}$ in the ES state.

In Fig. 2, a plot of the difference between the expected utilities versus $r$ is shown, where $r$ is the ratio of the number of players with $\mathrm{CW}_{\text {min }}^{\mathrm{L}}$ to the total number of players. Here $\alpha$ is fixed at a value of 3 . The difference is defined as

$$
h=f\left(\mathrm{CW}_{\min }^{\mathrm{L}}\right)-f\left(\mathrm{CW}_{\min }^{\mathrm{H}}\right),
$$

where $f(i)$ is the expected utility of a player with strategy $i$. Thus, $f\left(\mathrm{CW}_{\text {min }}^{\mathrm{L}}\right)$ and $f\left(\mathrm{CW}_{\text {min }}^{\mathrm{H}}\right)$ are calcurated as

$$
\begin{aligned}
f\left(\mathrm{CW}_{\min }^{\mathrm{L}}\right)= & u\left(\mathrm{CW}_{\min }^{\mathrm{L}}, \mathrm{CW}_{\min }^{\mathrm{L}}\right) r \\
& +u\left(\mathrm{CW}_{\min }^{\mathrm{L}}, \mathrm{CW}_{\min }^{\mathrm{H}}\right)(1-r),
\end{aligned}
$$

and

$$
\begin{aligned}
f\left(\mathrm{CW}_{\min }^{\mathrm{H}}\right)= & u\left(\mathrm{CW}_{\min }^{\mathrm{H}}, \mathrm{CW}_{\min }^{\mathrm{L}}\right) r \\
& +u\left(\mathrm{CW}_{\min }^{\mathrm{H}}, \mathrm{CW}_{\min }^{\mathrm{H}}\right)(1-r) .
\end{aligned}
$$

In Fig. 2, $r=r^{\star}(=0.66)$ corresponds to the ES state because $h=0$. When some players change their strategy and $r<r^{\star}$, $h>0$. In this case, the utility of the players with $\mathrm{CW}_{\text {min }}^{\mathrm{L}}$ is higher than that of the others. Therefore, the number of players with $\mathrm{CW}_{\min }^{\mathrm{L}}$ increases. Subsequently, $r$ returns to the original ES state, in which $r=r^{\star}$. The case where $r$ becomes greater than $r^{\star}$ can be discussed similarly.

A plot of the $r^{\star}$ versus $\alpha$ is shown in Fig. 3. As can

Table 6 Payoff matrix $u(i, j)$ when $\alpha=3$.

\begin{tabular}{|c|c|cc|}
\hline \multicolumn{2}{|c|}{} & \multicolumn{2}{|c|}{$j$} \\
\cline { 3 - 4 } \multicolumn{2}{|c|}{} & $\mathrm{CW}_{\min }^{\mathrm{L}}$ & $\mathrm{CW}_{\min }^{\mathrm{H}}$ \\
\hline \multirow{2}{*}{$i$} & $\mathrm{CW}_{\text {min }}^{\mathrm{L}}$ & -0.031 & 0.079 \\
& $\mathrm{CW}_{\min }^{\mathrm{H}}$ & -0.0096 & 0.038 \\
\hline
\end{tabular}

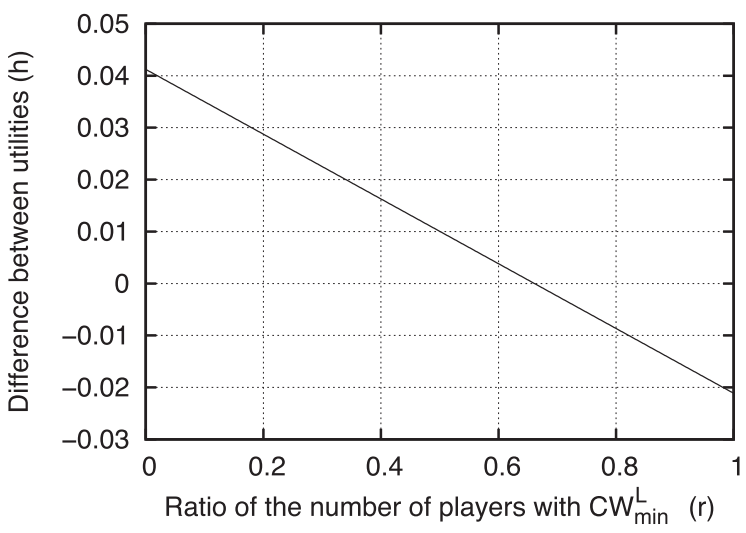

Fig. 2 Difference between the expected utilities vs. $r$. 


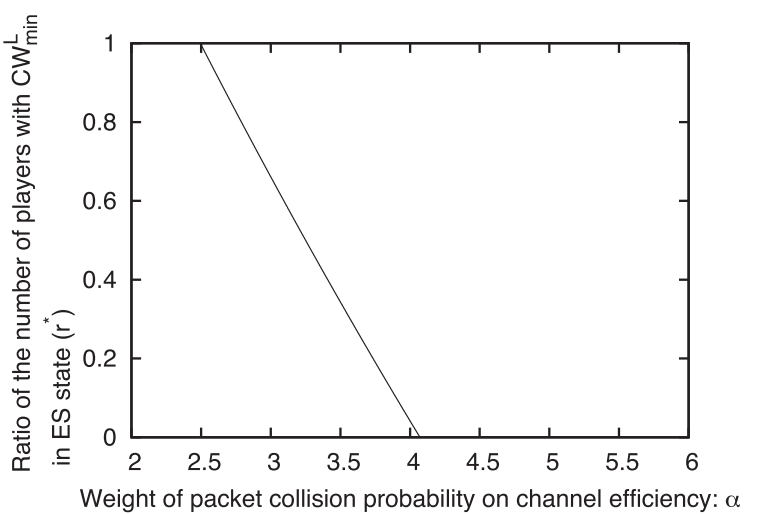

Fig. $3 r^{\star}$ vs. weight of packet collision probability on channel efficiency.

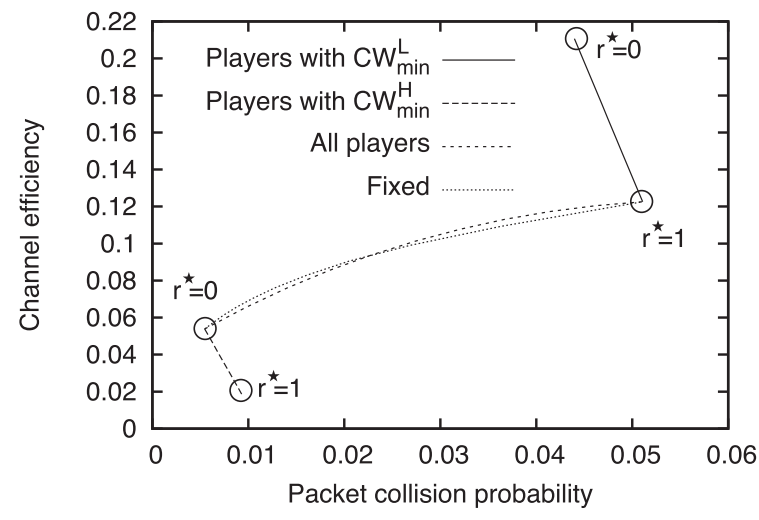

Fig. 4 Channel efficiency vs. packet collision probability.

be observed, $r^{\star}$ decreases monotonically. The increase in $\alpha$ implies that the importance of packet collision probability increases. In this system, the best way to avoid a packet collision is to increase the contention window size large. Therefore, $r^{\star}$ decreases. In Fig. 3, we can also see that the entire range of $r^{\star}$ values can be obtained by varying $\alpha$.

Using the entire range of $r^{\star}$, we show the impact of $r^{\star}$ on the channel efficiency and packet collision probability in Fig. 4. In this figure, the average channel efficiency and packet collision probability of players with $\mathrm{CW}_{\text {min }}^{\mathrm{L}}$, those of players with $\mathrm{CW}_{\min }^{\mathrm{H}}$, and those of all the players are shown respectively when $r^{\star}$ is varied from 0 to 1 . In addition, for a comparison, the channel efficiency and packet collision probability of fixed $\mathrm{CW}_{\min }$ scheme are shown in Fig. 4. In the fixed $\mathrm{CW}_{\min }$ scheme, only one $\mathrm{CW}_{\min }$ is used, and in this figure, the plot when $\mathrm{CW}_{\min }$ is varied from 15 to 127 is shown. We can see that the average channel efficiency and packet collision probability increase with $r^{\star}$. This observation is reasonable because an increase in $r^{\star}$ indicates a decrease in the average contention window size. In Fig. 4, we can also see that channel efficiency of players with $\mathrm{CW}_{\text {min }}^{\mathrm{L}}$ decreases when $r^{\star}$ increases. This is because the number of players with $\mathrm{CW}_{\min }^{\mathrm{H}}$ decreases and the packet collision probability increases when $r^{\star}$ increases. This increase in the packet collision probability causes the channel efficiency of players with $\mathrm{CW}_{\min }^{\mathrm{H}}$ to decrease. From the figure, we

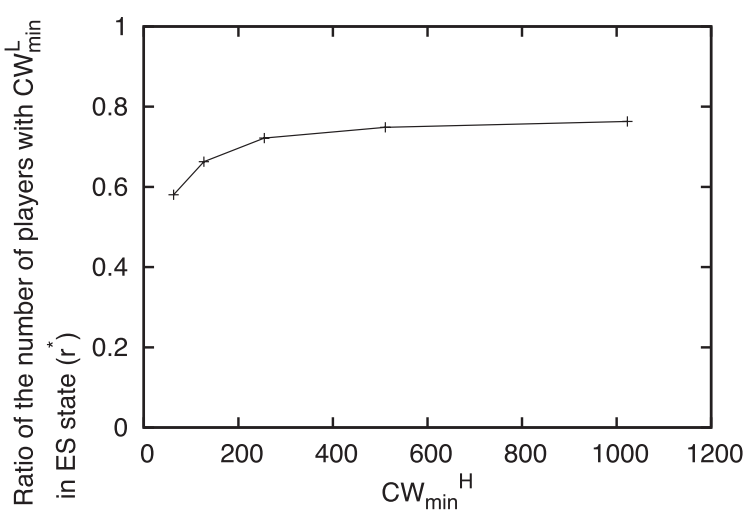

Fig. $5 r^{\star}$ vs. $\mathrm{CW}_{\min }^{\mathrm{H}}$.

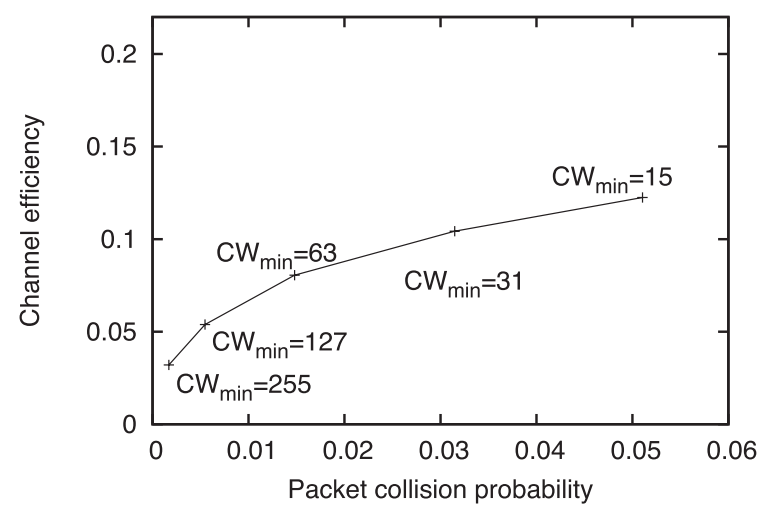

Fig. 6 Tradeoff for values of $\mathrm{CW}_{\min }$.

can also see that property of the fixed $\mathrm{CW}_{\min }$ scheme and property of all player in the proposed scheme are almost the same, although the property of player with each strategy is different largely. Using this property, it is expected that a QoS management can be achieved.

Figure 5 shows a plot of $r^{\star}$ versus $\mathrm{CW}_{\min }^{\mathrm{H}}$. Here $\alpha$ is fixed at a value of 3 . We can see that $r^{\star}$ increases throughout the plot. When $\mathrm{CW}_{\min }^{\mathrm{H}}$ increases, the average $\mathrm{CW}$ also increases. This leads to an increase in the backoff time. Therefore, the transmission opportunities decrease and the utility of players with $\mathrm{CW}_{\min }^{\mathrm{H}}$ also decreases. This leads to an increase in $r^{\star}$. Moreover, in Fig. 5, we can see that $r^{\star}$ saturates at a value of 0.8. In CSMA/CA, CW is doubled if a packet collision occurs. $\mathrm{CW}$ then reaches $\mathrm{CW}_{\max }$ after two or three packet collisions if $\mathrm{CW}_{\min }$ is large. This is the reason for the saturation.

To select $\mathrm{CW}_{\text {min }}^{\mathrm{L}}$ and $\mathrm{CW}_{\text {min }}^{\mathrm{H}}$, high channel efficiency and low packet collision probability are desirable. However, there is a tradeoff between channel efficiency and packet collision probability as can be seen in Fig. 6 in which the performances of some values of $\mathrm{CW}_{\min }$ is shown. For example, in Fig. 6, " $\mathrm{CW}_{\min }=15$ " means that all the mobile routers use $\mathrm{CW}_{\min }=15$. Thus, in the above analysis, the specific values of $\mathrm{CW}_{\text {min }}^{\mathrm{L}}=15$ and $\mathrm{CW}_{\text {min }}^{\mathrm{H}}=127$ are selected as an example and not the optimal values. 


\section{Learning Rules and Linear Conversion}

In this section, we discuss the convergence of the ratio of the number of players selecting a strategy to the total number of players. It is important to discuss the convergence because the network throughput depends on the convergence speed. In the evolutionary game theory, an ES learning rule and a relative payoff sum (RPS) learning rule are known as a method for strategy determination [5]. In this section, a threshold (TH) learning rule based on the ES and RPS learning rules is proposed. Further, we propose a linear conversion method for applying the learning rules to an evolutionary game in which the utilities have different signs.

\subsection{ES Learning Rule}

The ES learning rule has been proposed in [17]. In evolutionary game theory, there is a property that the strategy with which players get higher utility is preferred by players. The ES learning rule is based on the property. Each player selects a strategy with a probability that is calculated on the basis of the utilities that the player had in the past. Therefore, the state becomes ES. When the state is ES and the number of trials is quite large, player $k$ 's selection probability of strategy $i$ in trial $t$ can be written as

$$
g_{i}^{k}(t)=\frac{\sum_{\tau=1}^{t-1} v_{i}^{k}(\tau)}{\sum_{i=1}^{M} \sum_{\tau=1}^{t-1} v_{i}^{k}(\tau)},
$$

where $v_{i}^{k}(\tau)$ is the utility of player $k$ selecting strategy $i$ in trial $\tau$, and $M$ is the number of strategies. When player $k$ selects strategy $i$ in trial $\tau, v_{j}^{k}(\tau)=0(j \in\{1, \ldots, i-1, i+$ $1, \ldots, M\})$. This equation implies that the selection probability of a strategy with which a player can get a higher utility increases.

The validity of writing the selection probability in an ES state as (18) is shown by the following proof. Since it is assumed that $t$ is quite large and the state is the ES state, the probability can be written as

$$
g_{i}^{k}(t)=t_{i}^{k} / t
$$

where $t_{i}^{k}$ is the total number of trials when player $k$ select strategy $i$. Then, the expected utility of player $k$ upon selecting strategy $i$ can be written as

$$
E\left[v_{i}^{k}(t)\right]=\frac{1}{t_{i}^{k}} \sum_{\tau=1}^{t-1} v_{i}^{k}(\tau) .
$$

From the theorem of Bishop and Cannings, in an ES state,

$$
E\left[v_{i}^{k}(t)\right]=E\left[v_{j}^{k}(t)\right], \quad j, i \in\{1, \ldots, M\} .
$$

Then,

$$
E\left[v_{1}^{k}(t)\right]=\cdots=E\left[v_{M}^{k}(t)\right]=C,
$$

where $C$ is constant value. From (20) and (22), we can obtain

$$
\sum_{\tau=1}^{t-1} v_{i}^{k}(\tau)=C t_{i}^{k}
$$

From (19) and (23), we can obtain

$$
g_{i}^{k}(t)=\frac{1}{C t} \sum_{\tau=1}^{t-1} v_{i}^{k}(\tau)
$$

From $\sum_{i=1}^{M} g_{i}^{k}(t)=1$ and (24), we can obtain

$$
C t=\sum_{i=1}^{M} \sum_{\tau=1}^{t-1} v_{i}^{k}(\tau)
$$

From (24) and (25), we obtain (18).

\subsection{TH Learning Rule}

We can not apply the ES learning rule directly because the initial state is not assumed in the rule. To realize the ES learning rule, a RPS learning rule has been proposed in [17]. However, in the RPS learning rule, a preference coefficient is needed as a parameter: this parameter significantly affects the convergence point. For this reason, the RPS learning rule is not appropriate.

We propose the TH learning rule that does not need the preference coefficient. In the TH learning rule, player $k$ 's selection probability of strategy $i$ in trial $t$ can be written as

$$
g_{i}^{k}(t)=\left\{\begin{array}{l}
g_{i}(0), \quad t \leq T_{\text {th }} \\
\frac{\sum_{\tau=1}^{t-1} x^{t-1-\tau} v_{i}^{k}(\tau)}{\sum_{i=1}^{M} \sum_{\tau=1}^{t-1} x^{t-1-\tau} v_{i}^{k}(\tau)}, \quad t>T_{\text {th }},
\end{array},\right.
$$

where $T_{\text {th }}$ is a predefined threshold and $g_{i}(0)$ is the predefined selection probability of strategy $i$ satisfying

$$
\sum_{i=0}^{M} g_{i}(0)=1
$$

and

$$
g_{i}(0)>0 .
$$

A forgetting coefficient is denoted by $x(0 \leq x \leq 1)$. To increase the convergence speed, the forgetting coefficient is necessary. As the number of trials increases, the dependence of the utility that each player had in the past on the selection probability becomes stronger. This implies that the selection probability changes by a small amount when the number of trials is large. The convergence speed decreases as the state approaches to the ES state. To avoid a low convergence speed, the forgetting coefficient is needed.

When $t \leq T_{\text {th }}$, each strategy is selected with an equal probability. When $t>T_{\text {th }}$, each player selects a strategy on the basis of the utilities that the player has. In the TH learning rule, the convergence speed also depends on $T_{\mathrm{th}}$. This 
Table 7 Payoff matrix after linear conversion.

\begin{tabular}{|c|c|c|c|}
\hline & \multicolumn{2}{|c|}{$\bar{j}$} \\
\hline & & 1 & 2 \\
\hline$i$ & $\begin{array}{l}1 \\
2\end{array}$ & $\begin{array}{l}a+D \\
c+D\end{array}$ & $\begin{array}{l}b+D \\
d+D\end{array}$ \\
\hline
\end{tabular}

is because for a large $T_{\mathrm{th}}$, the sum of the utilities becomes large and the change in $g_{i}^{k}(t)\left(t>T_{\mathrm{th}}\right)$ becomes small. On the other hand, $T_{\text {th }}$ should have a considerably large value because there are unused strategies at $t \leq T_{\mathrm{th}}$ if $T_{\mathrm{th}}$ is too small. Consequently, the strategies are not selected at $t>T_{\text {th }}$ because the probabilities of the strategies are 0 . For this reason, $T_{\text {th }}$ should be large if the number of strategies is large.

\subsection{Linear Conversion}

We assume an evolutionary game where the payoff matrix is as shown in Table 2. From the table and (15), we obtain

$$
q=\frac{b-d}{c-a+b-d} .
$$

Here, we assume that the utilities have different signs. If we apply the learning rule, this difference leads to the condition $g_{i}^{k}(t)>1$ or $g_{i}^{k}(t)<0$. To remove the difference, we add a constant value, $D$, to all the utilities. In this way, we obtain Table 7. Using Table 7 and (15), we obtain

$$
q^{\prime}=\frac{b+D-d-D}{c+D-a-D+b+D-d-D}=\frac{b-d}{c-a+b-d} .
$$

From this equation, we can see that the ratio is the same as that obtained for the ES state. This is because the differences in the utilities are the same.

If $D$ increases, the convergence speed decreases. When $D$ is large, the ratio of the difference between the utilities to the average of the utilities is small. Further, the selection probability changes by an amount that is smaller than the amount when $D$ is small. This is the reason for the decrease in the speed.

\section{Evaluation of Dynamics}

We evaluate the dynamics of the system described in Sect. 2 by using the protocol proposed in Sect. 3. Each player uses the TH learning rule and decides upon a strategy.

We assume a fixed payoff matrix at $\alpha=3$. Then, the payoff matrix is as shown in Table 6. From the table and (15), we obtain

$$
r^{\star}=\frac{0.0079-0.038}{[(-0.0096)-(-0.031)]+0.0079-0.038}=0.66 .
$$

By applying the linear conversion to Table 6 and considering $D=0.031$, we obtain Table 8 .

We evaluate the dynamics by applying the $\mathrm{TH}$ learning rule in a computer simulation. In this simulation, the payoff
Table 8 Payoff matrix $u^{\prime}(i, j)$ when $\alpha=3.5$ and 0.28 is added to $u(i, j)$.

\begin{tabular}{|c|c|cc|}
\hline \multicolumn{2}{|c|}{} & \multicolumn{2}{|c|}{$j$} \\
\cline { 3 - 4 } \multicolumn{2}{|c|}{} & $\mathrm{CW}_{\text {min }}^{\mathrm{L}}$ & $\mathrm{CW}_{\min }^{\mathrm{H}}$ \\
\hline \multirow{2}{*}{$i$} & $\mathrm{CW}_{\min }^{\mathrm{L}}$ & 0.0 & 0.11 \\
& $\mathrm{CW}_{\min }^{\mathrm{H}}$ & 0.21 & 0.068 \\
\hline
\end{tabular}

Table 9 Simulation parameters.

\begin{tabular}{c|c}
\hline Parameters & Values \\
\hline Number of players & 200 \\
$T_{\text {th }}$ & 100 \\
Number of trials & $10^{6}$ \\
$x$ & 0.99 \\
\hline
\end{tabular}

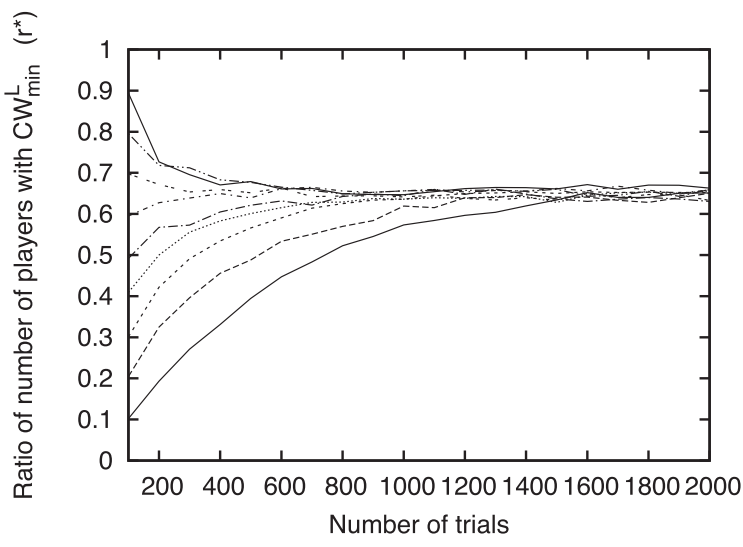

Fig. 7 Learning dynamics in TH learning rule.

matrix is fixed and is as shown in Table 8 after the linear conversion. From the table and (15), we obtain

$$
r^{\star}=\frac{0.11-0.068}{0.21-0.0+0.11-0.068}=0.66 \text {. }
$$

Each trial in the simulation is as follows.

1. Each player selects a strategy on the basis of the $\mathrm{TH}$ learning rule.

2. All the players are paired randomly. This means that every player is a part of a pair.

3. Players in each pair interact with each other and get utilities on the basis of the payoff matrix in Table 8 . Then, the selection probability is updated for each player, and the process returns to step 1 .

The simulation parameters and other parameters are listed in Tables 9 and 3, respectively.

Figure 7 shows the learning dynamics for the TH learning rule for $\alpha=3.5$. Then, we set $g_{\mathrm{CW}_{\text {min }}^{\mathrm{H}}}(0)$ to have values from 0.1 to 0.9 . From this figure, we can see that $r$ converges to $r^{\star}$. This is because the TH learning rule is almost identical to the ES learning rule when the number of trials is large.

\section{Conclusions}

MLANS randomly interact with each other because of their 
mobility. A system with such random interactions can be analyzed by using evolutionary game theory. We formulate the system as an evolutionary game. As a strategy selection, each mobile router selects the minimum contention window size from two values. We define the channel efficiency and packet collision probability as a utility, and the importance of the packet collision probability for the channel efficiency is predetermined. From the simulation results and analysis, we show that the ES state can be realized by varying the importance.

In addition, for a system of mobile routers, we propose a TH learning rule and a linear conversion that can be applied to the learning rule. Using the learning rule, each player selects a strategy based on the past trials. Then, we show the learning dynamics in the ratio of the number of players selecting a strategy. From the results, we confirm that the ratio converges to a value that denotes an ES state and that the state is stable.

\section{Acknowledgment}

This work is supported in part by a Grant-in-Aid for Young Scientists (B) (no. 21760287) and a Grant-in-Aid for Scientific Research (A) (no. 20246067).

\section{References}

[1] M. Luoto and T. Sutinen, "Cross-layer enhanced mobility management in heterogeneous networks," Proc. IEEE ICC 2008, pp.22772281, May 2008.

[2] P. Venkitasubramaniam, S. Adireddy, and L. Tong, "Sensor networks with mobile access: Optimal random access and coding," IEEE J. Sel. Areas Commun., vol.22, no.6, pp.1058-1068, Aug. 2004.

[3] EMOBILE, "Pocket Wifi (in Japanese)." http://emobile.jp/pocket wifi/

[4] SoftBank, "C01HW." http://mb.softbank.jp/en/products/data_com/ c01hw.html

[5] J. Smith, Evolution and the Theory of Games, Cambridge University Press, 1982.

[6] J. Hofbauer and K. Sigmund, "Evolutionary game dynamics," J. Bull. Amer. Math. Soc., vol.40, pp.479-519, 2003.

[7] D. Niyato and E. Hossain, "Dynamics of network selection in heterogeneous wireless networks: An evolutionary game approach," IEEE Trans. Veh. Technol., vol.58, no.4, pp.2008-2017, May 2009.

[8] M. Anastasopoulos, P.D. Arapoglou, R. Kannan, and P. Cottis, "Adaptive routing strategies in ieee 802.16 multi-hop wireless backhaul networks based on evolutionary game theory," IEEE J. Sel. Areas Commun., vol.26, no.7, pp.1218-1225, Sept. 2008.

[9] B. Hirantha Sithira Abeysekera, T. Matsuda, and T. Takine, "Dynamic contention window control mechanism to achieve fairness between uplink and downlink flows in ieee 802.11 wireless lans," IEEE Trans. Wirel. Commun., vol.7, no.9, pp.3517-3525, Sept. 2008.

[10] T. Yoshioka, S. Ohzahata, and K. Kawashima, "Fair bandwidth assignment to each terminal by adjusting contention window size in access point," Proc. IEEE Personal, Indoor and Mobile Radio Communications, 2009. PIMRC 2009, pp.762-766, Sept. 2009.

[11] M. Bottigliengo, C. Casetti, C.F. Chiasserini, and M. Meo, "Smart traffic scheduling in 802.11 wlans with access point," IEEE 58th Vehicular Technology Conference, 2003. VTC 2003-Fall, vol.4, pp.2227-2231, Oct. 2003.

[12] G. Bianchi, L. Fratta, and M. Oliveri, "Performance evaluation and enhancement of the csma/ca mac protocol for 802.11 wireless lans," Proc. IEEE Personal, Indoor and Mobile Radio Communications, PIMRC'96, pp.392-396, Oct. 1996.

[13] Y. Kwon, Y. Fang, and H. Latchman, "A novel mac protocol with fast collision resolution for wireless lans," Proc. IEEE INFOCOM 2003, pp.853-862, April 2003.

[14] "IEEE standard for information technology-telecommunications and information exchange between systems-local and metropolitan area networks-specific requirements - part 11: Wireless LAN medium access control (MAC) and physical layer (PHY) specifications," IEEE Std 802.11-2007 (Revision of IEEE Std 802.11-1999), pp.C11184, Dec. 2007.

[15] N. Nie and C. Comaniciu, "Adaptive channel allocation spectrum etiquette for cognitive radio networks," Mobile Netw. Appl., vol.11, no.6, pp.779-797, 2006.

[16] K. Yamamoto and S. Yoshida, "Game-theoretic approach to capacity and stability evaluations of decentralized adaptive route selections in wireless ad hoc networks," IEICE Trans. Commun., vol.E88-B, no.3, pp.1009-1016, March 2005.

[17] C.B. Harley, "Learning the evolutionarily stable strategy," J. Theoretical Biology, vol.89, no.4, pp.611-633, 1981.

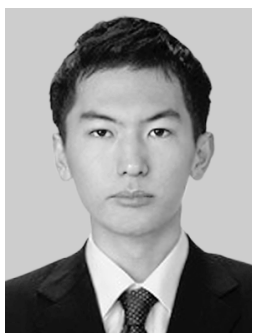

Ippei Aoki received the B.E. degree in electrical and electronic engineering from Kyoto University in 2009. He is currently studying towards his M.E. degree at the Graduate School of Informatics, Kyoto University. His research interests include game theory.

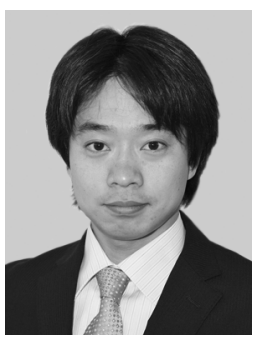

Koji Yamamoto received the B.E. degree in electrical and electronic engineering from Kyoto University in 2002, and the M.E. and Ph.D. degrees in informatics from Kyoto University in 2004 and 2005, respectively. From 2004-2005, he was a research fellow of the Japan Society for the Promotion of Science (JSPS). Since 2005, he has been with the Graduate School of Informatics, Kyoto University, where he is currently an Associate Professor. From 2008 to 2009, he was a visiting researcher at Wireless@KTH, Royal Institute of Technology (KTH) in Sweden. His research interests include game theory, spectrum sharing, and cooperative multi-hop networks. He received the PIMRC 2004 Best Student Paper Award in 2004, the Ericsson Young Scientist Award in 2006, and the Young Researcher's Award from the IEICE of Japan in 2008. He is a member of the IEEE. 


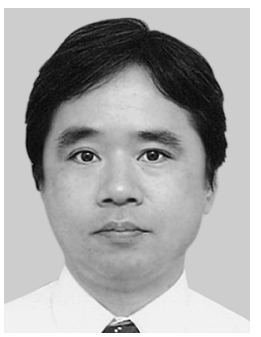

Hidekazu Murata received the B.E., M.E., and Ph.D. degrees in electronic engineering from Kyoto University, Kyoto, Japan, in 1991, 1993, and 2000, respectively. In 1993, he joined the Faculty of Engineering, Kyoto University. From 2002 to 2006, he was an Associate Professor of Tokyo Institute of Technology. He has been at Kyoto University since October 2006 and is currently an Associate Professor of Department of Communications and Computer Engineering, Graduate School of Informatics. His major research interests include signal processing and its hardware implementation, with particular application to cooperative wireless networks with cognitive radio capabilities. He received the Young Researcher's Award from the IEICE of Japan in 1997, the Ericsson Young Scientist Award in 2000, and the Young Scientists' Prize of the Commendation for Science and Technology by the Minister of Education, Culture, Sports, Science and Technology in 2006. He is a member of the IEEE and SITA.

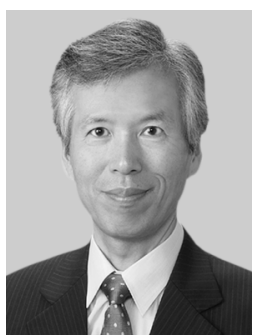

Susumu Yoshida received the B.E., M.E. and $\mathrm{Ph} . \mathrm{D}$. degrees all in electrical engineering from Kyoto University, Kyoto, Japan in 1971, 1973 and 1978, respectively. Since 1973, he has been with the Faculty of Engineering, Kyoto University and currently he is a full professor of the Graduate School of Informatics, Kyoto University. During the last 30 years, he has been mainly engaged in the research of wireless personal communications. His current research interest includes highly spectrally efficient wireless transmission techniques and distributed controlled wireless networks. During 1990-1991, he was a visiting scholar at WINLAB, Rutgers University, U.S.A. and Carleton University in Ottawa, Canada. He served as a TPC Chair of IEEE VTC 2000-Spring, Tokyo. He was a guest editor of IEEE J-SAC on Wireless Local Communications published in April and May 1996. He received the IEICE Achievement Award and Ericsson Telecommunication Award in 1993 and 2007, respectively. 